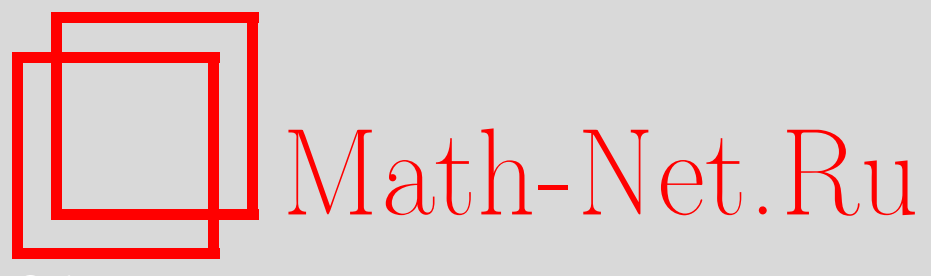

А. Д. Манита, Марковские процессы в непрерывной модели стохастической синхронизации, УМН, 2006, том 61, выпуск 5, 187-188

DOI: https://doi.org/10.4213/rm4947

Использование Общероссийского математического портала Math-Net.Ru подразумевает, что вы прочитали и согласны с пользовательским соглашением http://www . mathnet.ru/rus/agreement

Параметры загрузки:

IP: 52.90 .164 .192

26 апреля 2023 г., 13:50:43

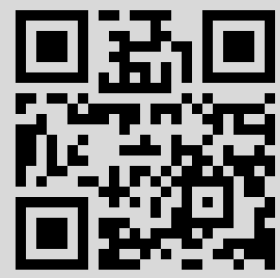




\section{Марковские процессы в непрерывной модели стохастической синхронизации}

\section{А. Д. Манита}

Мы рассматриваем некоторые классы марковских процессов со значениями в $C[a, b]$ и $D[a, b](a<b)$, которые мотивированы приложениями к стохастическим моделям параллельных и распределенных вычислений. Здесь $C[a, b]-$ нормированное пространство непрерывных функций $f:[a, b] \rightarrow \mathbb{R}$, а $D[a, b]$ - пространство функций, непрерывных справа и имеющих предел слева в каждой точке, топология в котором задается метрикой Скорохода [1]. В дальнейшем мы обозначим через $C_{0, m}[a, b]$ подмножество неубывающих функций $f \in C[a, b]$ с условием $f(a)=0$ и топологией, наследуемой из $C[a, b]$, а через $D_{+}[a, b]$ - подмножество неотрицательных функций из $D[a, b]$.

Пусть на вероятностном пространстве $(\Omega, \mathscr{F}, \mathrm{P})$ заданы следующие случайные последовательности: $0=\tau_{0}<\tau_{1}<\tau_{2}<\cdots$ - простейший (пуассоновский) поток интенсивности $\gamma$ и $\xi_{1}, \ldots, \xi_{n}, \ldots$ - независимые одинаково распределенные случайные величины, имеющие равномерно распределение в отрезке $[0,1]$. Предполагается, что последовательности $\left\{\tau_{i}\right\}$ и $\left\{\xi_{i}\right\}$ независимы между собой. Зафиксируем функцию $v \in$ $C^{1}[0,1], v(0)=0, v^{\prime}(x)>0$. Рассмотрим случайный процесс $\varkappa(t)=(\varkappa(t, x), x \in[0,1])$, $t \geqslant 0$, со значениями в $C_{0, m}[a, b]$, динамика которого по определению имеет две составляющие:

- Свободное движение: в интервалах между событиями пуассоновского потока $\left(\tau_{i}<\right.$ $\left.t<\tau_{i+1}\right)$ значения $\varkappa(t, x)$ изменяются с постоянной скоростью: $\frac{d}{d t} \varkappa(t, x)=$ $v(x)$;

- Случайные срезки: в моменты $\tau_{i}$ производится мгновенное преобразование функции ${ }^{1}: \varkappa\left(\tau_{i}+0, x\right)=\varkappa\left(\tau_{i}, x\right) \wedge \varkappa\left(t, \xi_{i}\right)$.

Другими словами, срезка заключается в том, что на участке $x \in\left(\xi_{i}, 1\right]$ профиль функции $\varkappa$ заменяется горизонтальным отрезком. Срезка соответствует синхронизации локальных времен в моделях параллельных вычислений. Всегда будем предполагать, что $\varkappa(0, x) \in C_{0, m}[a, b]$. Очевидно, что $\varkappa(t, 0)=0$ при всех $t$ и что множество $C_{0, m}[a, b]$ инвариантно относительно описанной динамики.

Легко видеть, что потраекторно (при каждом фиксированном $\omega$ ) имеет место представление $\varkappa(t, x)=\int_{0}^{x} \theta(t, r) d r$, где вспомогательный случайный процесс $\theta(t)=$ $(\theta(t, x), x \in[0,1])$ определен следующим образом. В интервалах $\tau_{i}<t<\tau_{i+1}$ между событиями пуассоновского потока значения $\theta(t, x)$ растут с постоянной скоростью: $\frac{d}{d t} \theta(t, x)=v^{\prime}(x)$; в моменты $\tau_{i}$ производится мгновенное преобразование $\theta\left(\tau_{i}\right)$ как функции от $x: \theta\left(\tau_{i}+0, x\right)=\theta\left(\tau_{i}, x\right) \cdot \chi\left(\xi_{i}-x\right)$, где $\chi(x)=\left\{\begin{array}{ll}1, & \text { если } x>0, \\ 0, & \text { если } x \leqslant 0 .\end{array}\right.$ Естественным множеством состояний для $\theta(t)$ является $D_{+}[0,1]$.

Зафиксируем $a \in(0,1)$ и каждой функции $\theta(t)=(\theta(t, x), x \in[0,1])$ поставим в соответствие ее сужение $\theta_{a}(t)=(\theta(t, x), x \in[a, 1])$ на отрезок $[a, 1]$. Введем случайный процесс

$$
\varkappa_{a}(t, x)=\int_{a}^{x} \theta_{a}(t, r) d r=\varkappa(t, x)-\varkappa(a, x), \quad x \in[a, 1] .
$$

В дальнейшем нас будут интересовать свойства одномерных распределений марковских процессов $\varkappa_{a}(t), \theta_{a}(t)$ и $\theta(t): \mu_{t}^{\varkappa, a}\left(M_{1}\right):=\mathrm{P}\left\{\varkappa_{a}(t) \in M_{1}\right\}, \mu_{t}^{\theta, a}\left(M_{2}\right):=$ $\mathrm{P}\left\{\theta_{a}(t) \in M_{2}\right\}$ и $\mu_{t}^{\theta}\left(M_{3}\right):=\mathrm{P}\left\{\theta(t) \in M_{3}\right\}$, где $M_{1} \in \mathscr{B}_{C_{0, m}[a, 1]}, M_{2} \in \mathscr{B}_{D_{+}[a, 1]}$,

Работа выполнена при поддержке РФФИ (гранты № 06-01-00662 и 05-01-22001).

${ }^{1} x \wedge y:=\min (x, y), x \vee y:=\max (x, y)$. 
$M_{3} \in \mathscr{B}_{D_{+}[0,1]}$, а $\mathscr{B}_{S}-\sigma$-алгебра борелевских множеств над метрическим пространством $S$. Следующая теорема устанавливает эргодичность марковских процессов $\varkappa_{a}(t)$ и $\theta_{a}(t)$.

Теорема 1. Существуют такие распределения $\mu_{\infty}^{\theta, a}$ на $\mathscr{B}_{D_{+}[a, 1]}$ и $\mu_{\infty}^{\varkappa, a}$ на $\mathscr{B}_{C_{0, m}[a, 1]}$, что при $t \rightarrow \infty$ имеют место сходимости по вариации:

$$
\mu_{t}^{\theta, a} \stackrel{\text { T.V. }}{\longrightarrow} \mu_{\infty}^{\theta, a}, \quad \mu_{t}^{\varkappa, a} \stackrel{\text { T.V. }}{\longrightarrow} \mu_{\infty}^{\varkappa, a} .
$$

ДокАзАтельство. Однородный марковский процесс $\theta_{a}(t)=(\theta(t, x), x \in[a, 1])$ со значениями в метрическом пространстве $D_{+}[a, 1]$ удовлетворяет условию Дёблина (см. [2; гл. $5, \S 5$, гл. $6, \S 2])$. Действительно, рассмотрим вложенную цепь Маркова $\Theta(n):=\theta\left(\tau_{n}\right) \in D_{+}[a, 1], n \in \mathbb{N}$. Из любого состояния эта цепь приходит в состояние $0 \in D_{+}[a, 1]$ с вероятностью $a: \forall \Theta_{0} \in D_{+}[a, 1] \mathrm{P}\left(\Theta(n)=0 \mid \Theta(n)=\Theta_{0}\right)=a>0$. Выполнение этого условия влечет геометрическую эргодичность марковской цепи $\Theta(n)$ и марковского процесса $\theta_{a}(t)$.

Рассмотрим отображение $\left(J_{a} g\right):=\int_{a}^{x} g(r) d r$. Оно действует из метрического пространства $D[a, 1]$ в метрическое пространство $C[a, 1]$ и является непрерывным. Очевидно, что $\varkappa_{a}(t)=J_{a} \theta_{a}(t)$. Таким образом, $\mu_{t}^{\varkappa, a}=\mu_{t}^{\theta, a} \circ J_{a}^{-1}$. Поскольку $\left\|\mu_{t}^{\theta, a} \circ J_{a}^{-1}-\mu_{\infty}^{\theta, a} \circ J_{a}^{-1}\right\|_{\mathrm{T} . \mathrm{V}} \leqslant\left\|\mu_{t}^{\theta, a}-\mu_{\infty}^{\theta, a}\right\|_{\text {T.V., }}$ мы получаем второе утверждение теоремы с $\mu_{\infty}^{\varkappa, a}=\mu_{\infty}^{\theta, a} \circ J_{a}^{-1}$.

Следующая теорема показывает, что многие свойства предельного распределения величин $\theta(t, x)$ для $x \in(0,1]$ могут быть получены в явном виде.

Теорема 2. Пусть $x \in(0,1]$. Предельное распределение случайной величины $\theta(t, x)$ экспоненциалъно: $\mathrm{P}_{\mu_{\infty}^{\theta}}\{\theta(x)>s\}=\exp \left(-\frac{\gamma x}{v^{\prime}(x)} s\right), s \geqslant 0$. В частности, $\mathrm{E}_{\mu_{\infty}^{\theta}} \theta(x)=\frac{v^{\prime}(x)}{\gamma x}, \mathrm{D}_{\mu_{\infty}^{\theta}} \theta(x)=\left(\frac{v^{\prime}(x)}{\gamma x}\right)^{2}$. Ковариачия и коэффичиент коррелячии равны соответственно $\operatorname{Cov}_{\mu_{\infty}^{\theta}}\left(\theta\left(x_{1}\right), \theta\left(x_{2}\right)\right)=\frac{v^{\prime}\left(x_{1}\right) v^{\prime}\left(x_{2}\right)}{\gamma^{2}\left(x_{1} \vee x_{2}\right)^{2}}, \rho_{\mu_{\infty}^{\theta}}\left(\theta\left(x_{1}\right), \theta\left(x_{2}\right)\right)=\frac{x_{1} \wedge x_{2}}{x_{1} \vee x_{2}}$.

Несмотря на то что $\varkappa_{a}(t, 0) \equiv 0$, предел при $t \rightarrow \infty$ распределения процесса $\varkappa(t)=(\varkappa(t, x), x \in[0,1])$, рассматриваемого как процесс со значениями в $C[0,1]$, может и не существовать. Как показывает следующая теорема, этот факт связан с поведением функции $v(x)$ в нуле, а достаточное условие существования такого предела чуть сильнее, чем условие $v^{\prime}(0)=0$.

Теорема 3. Для существования предела при $t \rightarrow \infty$ семейства распределений $\mu_{t}^{\varkappa}$, заданных на $\mathscr{B}_{C_{0, m}[0,1]}$, достаточно выполнения одного из двух эквивалентных условий: для некоторого $\delta>0$

$$
\int_{0}^{\delta} \frac{v(s)}{s^{2}} d s<\infty \quad \Longleftrightarrow \quad \int_{0}^{\delta} \frac{v^{\prime}(s)}{s} d s<\infty .
$$

Процесс $\varkappa(t)$ является непрерывной модификацией каскадной модели параллельных вычислений с субординацией [3], а $\varkappa(t, x)$ описывает макроскопическую динамику локального времени в узле $x$. Как следует из результатов [3], предположение $v^{\prime}(x)>0, x \in[0,1]$, означает, что в настоящей работе мы рассматриваем только одну синхронизованную группу (кластер) вычислительных устройств.

\section{Список литературы}

[1] П. Биллинсли, Сходимость вероятностных мер, Наука, М., 1977. [2] Дж. Л. Дуб, Вероятностные процессы, ИЛ, М., 1956. [3] А. Manita, F. Simonot, Stochastic Algorithms: Foundations and Applications, Third International Symposium (Moscow, 2005), Lect. Notes Comput. Sci., 3777, eds. O. B. Lupanov et al., 2005, 26-37.

А. Д. Манита (А. D. Manita)

Московский государственный университет им. М. В. Ломоносова

E-mail: manita@mech.math.msu.su
Представлено А. В. Булинским Принято редколлегией 01.08 .2006 